\title{
Learning from Interviews with HIV-Discordant Couples (Male Positive, Female Negative): The Challenges and Successes
}

\author{
Sandy N. Tecimer ${ }^{1,2}$, Denise Jaworsky ${ }^{1,2}$, Trent Newmeyer ${ }^{3}$, Steven Chihrin ${ }^{4}, K_{\text {Kevin Gough }}{ }^{2,5}$, Anita \\ Rachlis $^{2,6}$, James Martin ${ }^{7}$, Saira Mohammed ${ }^{1}$ and Mona R. Loutfy ${ }^{*}, 1,2,5$ \\ ${ }^{I}$ Women's College Research Institute, Women's College Hospital, Toronto, Ontario, Canada \\ ${ }^{2}$ University of Toronto, Toronto, Ontario, Canada \\ ${ }^{3}$ Brock University, St. Catharines, Ontario, Canada \\ ${ }^{4}$ University of Western Ontario, London, Ontario, Canada \\ ${ }^{5}$ St.Michael's Hospital, Toronto, Ontario, Canada \\ ${ }^{6}$ Sunnybrook Health Sciences Centre, Toronto, Ontario, Canada \\ ${ }^{7}$ Southern Ontario Fertility Technologies, London, Ontario, Canada
}

\begin{abstract}
This article examines the challenges and successes of recruiting participants and maintaining momentum in a small qualitative study on the experiences of HIV-discordant couples (where the male is HIV-positive and the female is HIV-negative) undergoing fertility assessment and/or treatment in Ontario, Canada, to reduce the risk of HIV transmission to the woman and fetus. The purpose of this article is to identify barriers and successes encountered in our study, consider how these are addressed in the literature, and highlight specific factors that need to be considered when studying a unique population similar to ours.
\end{abstract}

Keywords: HIV-discordant, fertility, methodology, recruitment.

\section{INTRODUCTION}

An estimated 66,000 individuals in Canada live with HIV, the vast majority of whom are of reproductive age [1]. As newer and more potent anti-HIV therapies have become available, the natural history of the virus has changed, resulting in prolonged life expectancy and an improved quality of life [2]. Naturally, the desire of people living with HIV to have children has also increased $[3,4]$. Historically, couples where at least one partner was infected with HIV have been discouraged from making plans to have children and many fertility clinics have refused to perform procedures for them [5]. In recent years, however, it has become increasingly accepted in the medical community to support these couples in having children [5] and there is a pressing need for further research involving this patient population. The fact that this population is doubly vulnerable (seeking fertility treatment and HIV-positive) and difficult to identify ("hidden") introduces a number of methodological challenges that need to be addressed.

In this paper, we analyse the challenges and successes encountered in a small, qualitative study of the experiences of couples where the male is HIV positive and the female is HIV negative (hereafter, HIV-discordant couples) seeking assistive reproductive technologies (ART) in Ontario,

*Address correspondence to this author at the Women and HIV Research Program, Women's College Research Institute, Women's College Hospital, 790 Bay St. Room 743, Toronto, ON, M5G 1N8, Canada, Tel: (416) 3513732, Ext. 2324; Fax: (416) 351-3746; E-mail: Mona.Loutfy@wchospital.ca
Canada. For such couples, the most widely used technology is sperm washing followed by intrauterine insemination (IUI). Since 1987, there have been over 3,600 published attempts to conceive using sperm washing in HIV-discordant couples, with no documented cases of HIV transmission [6]. However, little is known about these couples' experiences in Ontario. The results of the qualitative study will be reported elsewhere [7]. Here, we identify key challenges to undertaking research with this population - accessing and recruiting hidden and vulnerable populations, long distances between study sites and participants, and interviewing couples - which result from the small, hidden population and stigma and privacy issues of HIV-discordant couples seeking fertility treatment. Strategies for designing and executing recruitment and retention protocols are discussed at length.

Following a brief outline of our main study protocol and results, we describe the methods we used to overcome barriers in the 'Methods' section: a two-pronged approach to recruitment, the use of telephone interviews, adding a research team member close to the fertility/HIV clinics, and ensuring flexibility in conducting interviews. In the 'Challenges and Successes' section, we present a detailed discussion of the challenges in dealing with discordant couples seeking fertility treatment and the successes of our methods. Recommendations for future studies are given in the 'Discussion' section. 


\section{METHODS}

\section{Main Study Protocol}

In this study, we documented the experiences of Ontario HIV-discordant couples pursuing ART to reduce horizontal transmission risk, with the goal of identifying gaps in patient care. We recruited 14 HIV-discordant couples between 2007 and 2009 to participate in chart reviews and semi-structured interviews. One couple withdrew from the study and another couple participated only in the chart review, leaving 12 couples that were interviewed and 13 couples that had their chart reviewed. The research ethic boards of the participating sites approved the study and informed consent was obtained from all participants.

\section{Respondent Recruitment}

Inclusion criteria included couples that: 1) were at least 18 years old, 2) were HIV-discordant, where the female partner was HIV-negative and the male partner was HIVpositive, 3) were interested in conceiving, and 4) had visited a fertility clinic in Ontario to discuss sperm washing as a method to reduce horizontal HIV transmission. Recruitment was conducted through one of the only two clinics that offered sperm washing at the time in Ontario (both private clinics) as well as HIV care providers (from both academic and community practices) in Ontario. The second clinic offering sperm washing was also a study site, but no enrolment occurred through this site. Eligible HIVdiscordant couples were initially contacted by their fertility clinic and/or HIV care provider. Participants expressing interest were subsequently contacted by a research team member who provided additional study information, determined eligibility, obtained informed consent and conducted the interview.

\section{Chart Review Data}

Retrospective chart reviews were conducted on health care records maintained by the participants' HIV physicians and the participating fertility clinic, when available. Data collected via chart review were related to four areas for each couple: 1) demographics, 2) HIV history, 3) fertility history, and 4) other medical history. Data not found through chart reviews was collected through the interviews or a follow-up phone call.

\section{Qualitative Data Collection}

Qualitative data was collected through in-depth semistructured interviews with each couple, either in person or over the phone. In both cases, members of the couple were interviewed together. Questions were asked following a semi-structured interview guide, addressing the following areas: 1) desire to have children, 2) worries concerning conception, 3) feelings regarding fertility, 4) impact on lifestyle, 5) support networks, 6) physician counselling on pregnancy planning received, 7) sources of information on pregnancy planning in the context of HIV, 8) opinion on the current available support, and 9) satisfaction with fertility planning experience. Within each domain, participants were asked for recommendations of ways in which their experiences could have been improved. The interviews lasted between ninety minutes and two hours and were audio-recorded.

\section{Highlights of the Main Study Results}

All participants lived in Ontario and all but three were of Caucasian descent. The average age of female and male participants was 37 and 38 years, respectively. Four men were on permanent disability, receiving a settlement package offered by the government related to the acquisition of HIV through blood products. Eight of the men were haemophiliacs who received HIV infected blood products, 3 identified as men who have sex with men, and one was from a country with high HIV prevalence. The couples had a total 9 biological children together, of which 6 were conceived via sperm washing, one couple conceived naturally and two used donor insemination. In addition, 3 couples were pregnant at the time of interview, via sperm washing.

Five key themes emerged from the interviews: 1) motivations for pregnancy, 2) access, 3) knowledge, 4) support, and 5) stigma and secrecy. Participants were motivated to have children when it became clear newer medicines made it possible to live a "normal" life. Access to services was difficult, as very few clinics offered ART for HIV-discordant couples, they were not nearby and few health care providers (HCPs) or those living with HIV were aware of available options. Support varied amongst HCPs, family and friends, and also depended on whether couples disclosed their HIV status to others. Couples felt that to improve experiences there needs to be a greater number of fertility clinics offering ART services to people living with HIV and also that HCPs and AIDS service organizations should be far more knowledgeable about the procedure. It was noted that few HCPs asked directly about couples' family planning desires and that if HIV status was not disclosed to family and friends, HCPs may be the only source of support they have throughout the process.

\section{Methods Used to Overcome Recruitment and Retention Barriers}

\section{Two-Pronged Recruitment Strategy}

Discordant couples seeking fertility treatments make up a small and moreover, hidden, population due to stigma associated both with HIV status and the need for fertility treatments. In order to maximize recruitment, we adopted a two-pronged approach in which potential participants were recruited from: 1) one of the only two fertility centres in Ontario known to provide specific services to people living with HIV, and 2) from three large urban HIV clinics.

\section{Telephone Interviews}

In addition to offering to conduct face-to-face interviews with potential study participants, we offered telephone interviews. This allowed for the inclusion of potential participants who could not participate in face-to-face interviews due to travel barriers.

\section{Adding a Team Member at Clinic Sites}

In an attempt to address communication barriers between ourselves and clinic staff, in addition to regular phone and email contact, we introduced a team member located in the vicinity of one of the fertility clinics, whose responsibility it was to give a presentation, meet with staff and answer questions. This new member also served to conduct 
interviews with couples that lived closer to the fertility clinic.

\section{Interviewer Flexibility}

In order to overcome the concerns of distance and the potential problems associated with scheduling interviews with couples, we ensured that all interview schedules were very flexible for couples. Interviewers offered to schedule interviews during evenings, weekends and holidays. In addition, interviews could be held in locations both convenient and comfortable for the couples, such as at home, or at clinics.

\section{CHALLENGES AND SUCCESSES}

\section{Accessing and Recruiting a Hidden, Vulnerable Population}

\section{Challenge: Identifying Potential Participants}

A key challenge in conducting research on HIVdiscordant couples considering fertility treatment is that much of this population is hidden to researchers and health care providers. There are no formal databases of HIVdiscordant couples wishing to conceive via fertility treatment, and the number of known couples is small. As the use of ART as an option for HIV-discordant couples has been limited, few health care providers ask about fertility desires $[4,8]$. Many couples do not disclose their HIV status due to stigma, making it difficult to identify those who have tried to access ART options. As a result, much of this population is not readily accessible through standard recruitment channels, and traditional random sampling methods are not appropriate.

Given the absence of a sampling frame for the HIV population in Canada or Ontario, and the sensitive nature of the study, non-probability or purposive sampling was appropriate and gatekeeper referral was used to make initial contact. For confidentiality reasons we-as research personnel-could not participate in the recruitment of potential participants at the fertility or HIV clinics. This created several challenges: 1) communication barriers between ourselves (the research personnel) and clinic staff delayed recruitment, 2) the fertility clinic did not have staff with salaried time protected for research, requiring them to assist with the study when they had additional time between clinical duties, and 3) clinical staff were put in a position where they had to balance the privacy of their clients with research interests. Indeed, the gatekeepers involved in our study often expressed concerns regarding the privacy of patients.

Access to research participants can be prevented or significantly delayed if gatekeepers are not adequately involved in the research process. Therefore, a crucial aspect of successful recruitment is the development of a strong relationship between researchers and gatekeepers [9]. Researchers often have a vested interest in the study well before recruitment staff is involved: they conduct literature reviews, formulate hypotheses, develop the study design, and often apply for funding opportunities. Thomas [9] reflected on his team's experiences between research staff and gatekeepers (study site therapists), identifying the importance of respecting the gatekeepers' unique cultures, recognizing their concerns, allowing and encouraging them to collaborate in study design to increase their sense of ownership, and ensuring they understand how they and their clients will benefit from the research. Witte et al. [10] also noted the benefits of taking the initiative to encourage closer relationships between researchers and gatekeepers.

\section{Successes}

In order to access as many potential participants as possible, we utilized a two-pronged approach to recruitment, recruiting through both the primary HIV physician in addition to fertility clinic staff. While fertility clinics do not usually remain in regular contact with clients after treatment, they have a greater number of eligible participant couples than the HIV clinics since every HIV-discordant couple seeking ART must contact them at some point to receive services. Furthermore, our research found that not all HIV physicians ask their patients about fertility desires. Hence, we anticipated that the majority of our recruits would come to us from fertility clinics, and the HIV clinics were involved only to cast as wide a net as possible. A surprising finding of our study was that it was actually the HIV clinics that made initial contact more often. This may be due to factors including more frequent medical visits with eligible couples, an active interest in academic research, a greater capacity to allocate resources to the project, or, more likely, a combination of these factors.

Before the introduction of an additional team member to act as a liaison and improve communication between the study and the research staffs, there was no recruitment via the fertility clinic. The addition of this liaison gave us someone who was very familiar with the study protocol to meet with staff members at the clinic, explain the purpose of the study, answer questions and be a contact person for them. This made it easier for staff to voice their concerns and increased the rate at which potential participants were identified and contacted. On the basis of discussions with fertility clinic staff members upon completion of the study, we conclude that more could have been done to improve the crucial gatekeeper-researcher relationship. Strategies for doing this are addressed in the Discussion.

\section{Challenge: Recruiting a Vulnerable Population}

Once potential participants (couples who expressed interest in participating in the study) were identified by the HIV care providers and/or fertility clinic, they were contacted by a member of the research team to receive more information. This aspect of recruitment represented a barrier for many potential participants due to the stigma associated both with living with HIV and pursuing fertility treatments (rendering them "doubly-vulnerable"). Early on in the study, we learned that some couples did not disclose their HIV status to other family members or friends. Furthermore, they did not share with others that they were undergoing fertility treatments for fear that this would reveal their HIV status. When family members or friends had asked them why they were having fertility treatments, they felt forced to either answer the question vaguely or create false reasons to prevent inadvertent HIV disclosure. The desire for discretion concerning fertility treatments was especially prominent in couples where the perceived suspicion of having HIV was already present, for example among men who were known by friends and family to be haemophiliac. Thus, we tried to 
be as sensitive as we could in our approach to making contact with potential participants. This may have been an undetected but significant barrier to recruitment, since communication with potential participants in the course of recruitment was vague and recipients may have had difficulty discerning the purpose of the communication.

The stigma of living with HIV has long been considered a great obstacle in treatment and prevention success, impacting testing and treatment uptake, and access to services $[11,12]$. Fertility treatments are clearly affected by this stigma, as evidenced by the limited services available to people living with HIV [13-16]. Furthermore, the limited available data describing the experience of those undergoing such treatments suggest that stigma is a major factor for these couples, both in terms of accessing options for having children, as well as the process of undergoing treatments without identifying to others why they are seeking fertility services [14].

\section{Successes}

Researchers contacting potential participants took every reasonable precaution to ensure privacy and disguised the reasons for calling from everyone except the potential participants. Our careful approach was successful in maintaining confidentiality and at no point during recruitment was any information passed inadvertently to the wrong party. Despite this, as noted above, the necessary vagueness required in calling potential participants at home may have acted as an additional barrier to recruitment. Some strategies for circumventing this are outlined in the Discussion.

\section{Distances Travelled by Participants and Researchers}

\section{Challenges}

Another challenge in this study was related to the disparate geographic locations of study site staff, researchers, participants and subsequent long travel distances required for the interviews. This issue is particularly acute for a small patient population such as ours: During study enrolment, only two fertility centres in Canada offered fertility services to HIV-discordant couples. As both were located in southern Ontario, those interested in sperm washing would have to travel to one of these centres to access this service. In addition, since access to HIV care tended to be concentrated in larger urban settings, many potential participants from more rural areas travelled to urban centres for HIV care. Our solution to this problemenhanced flexibility on the part of interviewers and the use of telephone interviews-introduced its own set of challenges. Advantages to telephone interviewing include lower costs, greater access to people living far away, less space requirements, ability to take notes unobtrusively, greater anonymity, ability for participants to be comfortable in their environment, potential increased safety for interviewers and participants, and potential for better facilitation of disclosure of more sensitive information $[17,18]$. Disadvantages to telephone interviews include lack of telephone access for some participants, lack of visual cues, potential distractions, and potentially shorter interview times. Novick [18] suggests that there is bias against telephone interviews among researchers, as even researchers who found phone interviews to be effective in their studies suggested that the telephone was a good second choice to face-to-face interviews.

\section{Successes}

We found that flexibility had a positive effect on recruitment and retention, as we had great success in recruiting participants who would otherwise have had difficulties meeting with us. Our experience with telephone interviews (three couples) was very positive as the couples who chose this mode felt it was much more convenient for them. They thought it was easier than meeting in person and were often interviewed after they had put their children to bed. One drawback we encountered was technical difficulties with recording the phone interviews, which may have affected the quality of data collection; however, this problem improved as our experience with telephone interviews increased.

\section{Interviewing Couples}

\section{Challenges}

Fertility treatments such as sperm washing and IUI are an inherently couple-oriented process. The key question that arises in interviewing couples for a research study is whether it is better to interview each member of the couple alone, interview them together, or interview them individually and then together as a couple. The benefits of interviewing couples (or other key stakeholders) together include retrieving more comprehensive data, allowing the couple to fill in one another's gaps, and observation of the dynamics of the relationship [19]. The couples interviewed together can use each other to recall forgotten stories and spark spontaneous discussion, allowing more information to be recalled and explored [20]. It can allow for the identification of discrepancies between each individual's memory of events, and determine their different perceptions of their stories $[19,20]$. A joint interview may give a more reliable and comprehensive picture than either member's individual account since a bias in one direction may be balanced by another version from the partner [21].

While disagreements between a couple's accounts of a situation or philosophy in a joint interview may allow for a better understanding of their ideas by allowing the interviewer to explore these differences in depth with them, there can be significant disadvantages to interviewing couples together. It may bring to the surface tensions in the couple that may persist well after the interview concludes $[19,20]$. Participants provide consent to participate in an interview, but neither they, nor the interviewer, can anticipate what sort of information, "forgotten and well hidden resentments or past half-truths", may emerge in the process, nor their impact on the relationship [22]. This should be considered for sensitive topics and if couples are to be interviewed together, the risk of creating tensions should be addressed in the informed consent process [22]. There is the chance that an individual may withhold details or feel uncomfortable in the presence of the other member. There is also the phenomenon of trying to present a "united front" [20], wherein a story is adjusted to avoid giving evidence of relationship discord, or to prevent giving what they feel is a negative perception of their relationship. There 
is also a concern that if one person dominates the interview, the experience may not have the richness of two accounts of the same events [19]. Some authors have found that individual interviews may facilitate more discussion on sensitive matters [21], and there is the consideration that in sensitive situations couples may refuse to participate in the study when they are asked to be interviewed together.

There is no consensus in the literature of what is the best way to interview couples (or household members): together, alone or both. When addressing the advantages and disadvantages of the various approaches to interviewing, authors should think carefully about their research area and identify which method is appropriate for their study while addressing logistical concerns [19-23]. In the context of our study, we felt it was important to be consistent in interviewing all couples in the same manner and, due to the highly sensitive nature of the study, wanted to reduce the risk of exposing "secrets" to the other partner if interviews were conducted separately or separately and then together. Furthermore, since the goal of the study was to document the joint experience of couples, the decision was taken to interview couples together. Even in this situation, however, the possibility of secrets being exposed during the interview process still existed. Thus, we made it clear from the outset (both on the information sheet and during the informed consent process) that if there were topics which they did not want raised, or were very sensitive between them as a couple, they should consider very carefully whether the study was a good idea for them.

\section{Successes}

Couples were interviewed together with the understanding that they would be able to end the interview at any time, refuse to answer particular questions, or inform us of feelings of discomfort with a line of questioning or a topic. Each couple-including one that had separated-was comfortable being interviewed together and the interviews proceeded very smoothly. While this worked very well for our study, it is possible that by restricting ourselves to only interviewing couples together, we may have lost access to some information that individuals did not want revealed to their partner, or prevented potential participants from joining because of this criterion. Most of the couples in our study reported a very positive experience with their sperm washing fertility services, but it is possible that couples with negative experiences or significant intra-couple conflict chose to not participate, resulting in sampling bias. For a better understanding of these couples' experiences, future studies require a broader sample population.

\section{DISCUSSION}

In the preceding sections, we have described in detail the challenges and successes in conducting research with a hidden, vulnerable population: HIV-discordant couples seeking fertility treatment. While the strategies described in Methods were largely successful in dealing with these challenges, there are a number of areas where future studies could benefit from additional efforts. These are outlined below.

Our two-pronged approach to recruitment (using health care-providers at fertility and HIV clinics) led to some problems. Inevitably, some participants were contacted twice, an unavoidable consequence of ensuring confidentiality since communication between clinics about participant recruitment would represent a breach of confidentiality. This was an issue for at least one potential participant who complained to the fertility clinic staff that a "second attempt" at recruitment, after they had already declined once, was not appropriate. The negative impact of approaching potential participants twice for the same study could have been mitigated by explaining (with each contact attempt) that the fertility or HIV clinic might also contact them to gauge their interest in the study. We should have clearly explained that the purpose of a second call was not to try to recruit them for a second time but that, in order to respect their privacy, the clinics could not communicate with one another about potential participants unless they had consented to participate.

During the study we found that many participants learned of fertility services through haemophiliac organizations or haematologists, and in hindsight, we may have been able to increase recruitment by accessing potential participants through these groups. Our study relied entirely on gatekeepers-HIV and fertility clinic staff-to identify potential participants. MacDougall and Fudge [24] have suggested that researchers should not depend solely on gatekeepers to recruit participants, but also look for other means to continue enrolment, such as snowball sampling or advertising directly to potential participants, for example through posters or flyers. At the conclusion of our study, it became clear to us that in addition to improving our communication with clinic staff, we should have tried to recruit using as many approaches as possible, including snowball sampling and direct advertising via community newsletters and to patient support groups.

Snowball sampling is a common, albeit criticized, approach to recruit hard-to-reach participants, whereby an eligible participant refers the researcher to another potentially eligible participant [25]. The idea is that there is a higher level of access to a specific population by other people in the same situation through the sharing of friends, support services, etc. [26]. Snowball sampling requires high levels of trust between the researcher and research participant. In our study, it became obvious that this recruitment method could have been a successful tool, as several participants asked if they could tell their friends, who were potential participants, about the study. We caution, however, that there are problems with this approach stemming from its intrinsic biases, especially in marginalized and hidden populations [26]. Encouragingly, there are efforts to increase the validity of snowball-sampling recruiting by modifying the process to reduce the biases intrinsic to the chain-referral approach. Such efforts include respondentdriven sampling [27,28], adaptive sampling [29], targeted sampling [30], or combinations of these techniques.

A common concern we encountered from our gatekeepers was the privacy of their clients; they expressed concern over how their clients' privacy may be violated by participating in research. Several strategies could have been used to alleviate this concern. Clinic staff could be thoroughly educated on the role of the research ethics boards and the responsibility of researchers in protecting the privacy and interests of research participants, thereby putting clinic 
staff more at ease. It is imperative that the site staff be aware of the goals of the research project, the motivation to conduct the study and the potential benefits participants may experience from the research [9]. If they have a clear sense of how the research can benefit their clients, they are likely to be more comfortable contributing to the research process.

Despite the addition of an extra staff member near one of the fertility clinics, it became clear to us at the end of the study that we had not sufficiently engaged the clinic staff as supporting stakeholders in the research study. The fertility clinic serves about 800 clients per year and, although a private clinic, conducts research regularly. The sensitive and personal nature of this study may have been novel for the site staff, however. At the end, we discovered there was still some uncertainty regarding the study among the fertility clinic staff, indicating that we failed to build a strong relationship with them. For future studies, we would recommend regular in-person meetings between study personnel and clinic staff to discuss recruitment challenges, provide positive feedback, and communicate protocol amendments. In addition, had we involved the clinic staff from the beginning of the research process, starting with the formulation of the research question and study design, we may have increased their sense of ownership in the project and also contributed to building research capacity.

The fact that the fertility clinics did not have dedicated research personnel meant that clinical staff had to assist us while also attending to full time clinical responsibilities. Competing demands on the time of clinic staff may make research-oriented activities a lesser priority than the more pressing clinical duties. Providing funding to the sites to compensate the clinic staff for their time would have been helpful to ensure that there was dedicated time protected for research activities.

As discussed earlier, a major issue when dealing with a doubly-vulnerable population such as the population of this study is the associated stigma and the crucial need to ensure that the privacy of participants is protected and that the participants are confident that this will happen in practice. Researchers working with vulnerable people have suggested overcoming such barriers with techniques that include: repeatedly ensuring confidentiality, building trust, understanding the specific cultural considerations of people who may join a study, presenting the study in a clear manner that will help potential participants and their families understand how they can benefit from participating, and reinforcing participants' altruistic intents [31,32]. Identifying the issues of concern to a participant prior to the start of a study and strategizing the resolution of these issues may significantly increase acceptance among participants, family members, relevant agencies and ethical review boards [31-33].

To ensure confidentiality and maximum privacy, we should have conveyed some of these sensitivities in the initial telephone contact. Having the clinic site staff ask the following questions prior to research staff contact would have been helpful:

1. What is the best way to contact you (e.g. phone number, time of day)?
2. Is it okay for the interviewer to leave details on your voicemail or with whoever answers the phone (e.g. that they are calling about a research study)?

3. Can the interviewer call back and leave other messages if there is no response to the first message or call?

A further significant problem in interviewing couples together that we failed to anticipate arose from the fact that many couples already had small children who required childcare during the interview. This is a problem that is not uncommon when interviewing young couples interested in starting or expanding their families, and as such is important in considering when designing a study. In one interview the children were present, and in another they were upstairs and periodically interrupted the interview, requiring the attention of a parent for brief periods. There was always the potential for the interview to be stopped early due to childcare needs or for participants to restrict their discussion for fear of being overheard by their children. Other couples had to find childcare for the duration of the interview. It would have been a significant help had we offered childcare or appropriate financial compensation.

\section{CONCLUSION}

Undertaking research involving a vulnerable, hidden population involves numerous challenges. In this paper, we document the challenges and successes of our recruitment and retention efforts in a small qualitative study designed to identify gaps in the care of HIV-discordant couples seeking fertility treatment in Ontario, Canada. Major barriers we identified included accessing a hidden and vulnerable population, the role of gatekeepers, long distances, issues pertaining to the interviewing of couples, and stigma and confidentiality in the face of sensitive issues.

We identified the major barriers encountered in our study, outlined strategies used to overcome them and finally made suggestions for adapting the study design to further enhance recruitment.

We hope that the discussion and recommendations given in this paper will be useful in the recruitment and retention of participants in qualitative studies, particularly in the context of HIV and fertility in order to further identify areas requiring systemic change to improve couples' experiences. By carefully documenting the challenges and successes of our qualitative study protocol and offering these recommendations, we hope that future studies may progress with greater efficiency.

\section{ACKNOWLEDGEMENTS}

We wish to thank all the couples who participated in the main study, sharing their experiences and time without compensation. This study would not have been possible without their openness and willingness to share private and personal details of their lives. We also would like to thank Maria Isabel Suarez, Linda Moran, Maureen McKenzie, and Pat McCabe for helping to recruit and contact participants. We are grateful to Dr. Emma Louise Clarence for assisting with editing the manuscript. We wish to thank the Women and HIV Research Program, Women's College Research Institute, Women's College Hospital, Toronto, Ontario, 
Canada for financial support for the transcriptions in this study.

\section{REFERENCES}

[1] HIV and AIDS in Canada selected surveillance tables to June 30, 2008. Available from: http://www.phac-aspc.gc.ca/aids-sida/publi cation/survreport/2008/pdf/survrep0608.pdf (Accessed January 3, 2011).

[2] Lima VD, Hogg RS, Harrigan PR, et al. Continued improvement in survival among HIV-infected individuals with newer forms of highly active antiretroviral therapy. AIDS 2007; 21: 685-92.

[3] Chen JL, Phillips KA, Kanouse DE, Collins RL, Miu A. Fertility desires and intentions of HIV-positive men and women. Fam Plann Perspect 2001; 33: 144-52.

[4] Panozzo L, Battergay M, Friedl A, Vernazza PL. High risk behaviour and fertility desires among heterosexual HIV-positive patients with serodiscordant partner-two challenging issues. Swiss Med Wkly 2003; 133: 124-7.

[5] Thornton AC, Romanelli F, Collins JD. Reproduction decision making for couples affected by HIV: a review of the literature. Top HIV Med 2004; 12: 61-7.

[6] Bujan L, Hollander L, Coudert M, et al. Safety and efficacy of sperm washing in HIV-1-serodiscordant couples where the male is infected: Results from the European CREAThE network. AIDS 2007; 21: 1909-14.

[7] Newmeyer T, Tecimer S, Jaworsky D, et al. Case series of fertility treatment in HIV-discordant couples (male positive, female negative): The Ontario experience. Forthcoming 2011.

[8] Sherr L, Barry N. Fatherhood and HIV-positive heterosexual men. HIV Med 2004; 5: 258-63.

[9] Thomas V. Conducting research with community agencies: Meeting recruitment and collaboration challenges. J Marital Fam Ther 2002; 28: 9-14.

[10] Witte SS, El-Bassel N, Gilbert L, Wu E, Chang M, Steinglass P. Recruitment of minority women and their main sexual partners in an HIV/STI prevention trial. J Women's Health 2004; 13: 1137-47.

[11] Lee RS, Kochman A, Sikkema KJ. Internalized stigma among people living with HIV-AIDS. AIDS Behav 2002; 6: 309-19.

[12] Brown L, Macintyre K, Trujillo L. Interventions to reduce HIV/AIDS stigma: What have we learned? AIDS Educ Prev 2003; 15: 49-69.

[13] Stanitis JA, Grow DR, Wiczyk H. Fertility services for human immunodeficiency virus-positive patients: provider policy, practice, and perspectives. Fertil Steril 2008; 89: 1154-8.

[14] Sunderam S, Hollander L, Macaluso M et al. Safe conception for HIV discordant couples through sperm-washing: Experience and perceptions of patients in Milan, Italy. Reprod Health Matters 2008; 16: 211-9.
[15] Wilde J. Conception in HIV-discordant couples. $2^{\text {nd }}$ ed. Treatment of Hemophilia April 2008; no. 26. Available from: http://www.wfh.org/2/docs/Publications/HIV_HepC/TOH-26-HIVdiscordant-couples-Revised2008.pdf (Accessed January 3, 2011).

[16] Yudin M, Shapiro M, Loutfy M. Advanced reproductive technologies for HIV-positive individuals and couples in Canada. Fertil Steril 2008; 90: S380.

[17] Carr ECJ, Worth A. The use of the telephone interview for research. Nursing Times Res 2001; 6: 511-24.

[18] Novick G. Is there a bias against telephone interviews in qualitative research? Res Nurs Health 2008; 34: 391-8.

[19] Arskey H. Collecting data through joint interviews. Soc Res Update 1996. Available from: http://sru.soc.surrey.ac.uk/SRU15.ht $\mathrm{ml}$ (Accessed January 3, 2011).

[20] Valentine G. Doing household research: interviewing couples together and apart. Area 1999; 31: 67-74.

[21] Racher FE, Kaufert JM, Havens B. Conjoint research interviews with frail, elderly couples: Methodological implications. J Fam Nurs 2000; 6: 367-79.

[22] Bailey C. Geographers doing household research: Intrusive research and moral accountability. Area 2001;33: 107-10.

[23] Beitin B. Qualitative research in marriage and family therapy: Who is in the interview? Contemp Fam Ther 2008; 30: 48-58.

[24] MacDougall C, Fudge E. Planning and recruiting the sample for focus groups and in-depth interviews. Qual Health Res 2001; 11: $117-26$

[25] Atkinson R, Flint J. Accessing hidden and hard-to-reach populations: Snowball research strategies. Soc Res Update 2001. Available from: http://sru.soc.surrey.ac.uk/SRU33.html (Accessed January 3,2011 ).

[26] Faugier J, Sargeant M. Sampling hard to reach populations. J Adv Nurs 1997; 26: 790-7.

[27] Heckathorn DD. Respondent-driven sampling: a new approach to the study of hidden populations. Soc Problems 1997; 44: 174-99.

[28] Heckathorn DD. Respondent-driven sampling II: Deriving valid population estimates from chain-referral samples of hidden populations. Soc Problems 2002; 49: 11-34.

[29] Thompson SK, Collins LM. Adaptive sampling in research on riskrelated behaviors. Drug Alcohol Depend 2002; 68: S57-67.

[30] Peterson J, Schacht RH, Schwartz RP, et al. Targeted sampling in drug abuse research: A review and case study. Field Methods 2008; 20: $155-70$

[31] Baker S. Black populations, human immunodeficiency virus/acquired immune deficiency syndrome, and research: implications for nurses. Appl Nurs Res 2001; 14: 94-9.

[32] Sherman DW, McSherry CB, Parkas V, Ye XY, Calbrese M, Gatto M. Recruitment and retention in a longitudinal palliative care study. Appl Nurs Res 2005; 18: 167-77.

[33] Moore LW, Miller M. Initiating research with doubly vulnerable populations. J Adv Nurs 1999; 30: 1030-40.

(C) Tecimer et al.; Licensee Bentham Open.

This is an open access article licensed under the terms of the Creative Commons Attribution Non-Commercial License (http://creativecommons.org/licenses/by-nc/ 3.0/) which permits unrestricted, non-commercial use, distribution and reproduction in any medium, provided the work is properly cited. 